Supporting Information

\title{
Water-Mediated Photochemical Treatments for Low-Temperature Passivation of Metal-Oxide Thin-Film Transistors
}

Jae Sang Heo ${ }^{1}$, Jeong-Wan Jo ${ }^{1}$, Jingu Kang ${ }^{1}$, Chan-Yong Jeong ${ }^{1}$, Hu Young Jeong ${ }^{2}$, Sung Kyu Kim ${ }^{3}$, Kwanpyo Kim ${ }^{4}$, Hyuck-In Kwon ${ }^{1}$, Jaekyun Kim ${ }^{5}$, Yong-Hoon Kim ${ }^{6}$, Myung-Gil $\mathrm{Kim}^{7^{*}}$, and Sung Kyu Park ${ }^{1^{*}}$

${ }^{1}$ School of Electrical and Electronics Engineering, Chung-Ang University, Seoul 156-756, Korea

${ }^{2}$ UNIST Central Research Facilities, Ulsan National Institute of Science and Technology (UNIST), Ulsan 689-798, Korea

${ }^{3}$ Department of Materials Science and Engineering, Korea Advanced Institute of Science and Technology (KAIST), Daejeon 34141, Korea

${ }^{4}$ Department of Physics, Ulsan National Institute of Science and Technology (UNIST), Ulsan 689-798, Korea

${ }^{5}$ School of Advanced Materials Science and Engineering, Hanbat University, Daejeon 305719 , Korea

${ }^{6}$ School of Advanced Materials Science and Engineering, Sungkyunkwan University, Suwon, Korea

${ }^{7}$ Department of Chemistry, Chung-Ang University, Seoul, Korea

Corresponding authors

*E-mail: myunggil@cau.ac.kr (M.-G.K.) and skpark@cau.ac.kr (S.K.P) 


\section{Table of Contents}

1. Table S1. Composition analysis of $a$-IGZO films with different annealing conditions.

2. Figure S1. Electrical characterization of DUV-irradiated $\mathrm{AlO}_{\mathrm{x}}$ dielectric layer.

3. Table S2. The summarized electrical properties of DUV-irradiated $\mathrm{AlO}_{\mathrm{x}}$ dielectric layer.

4. Figure S2. The transfer characteristics of DWD-annealed $a$-IGZO TFTs.

5. Figure S3. The statistical distribution of hysteresis voltages.

6. Figure S4. The threshold voltage shift of solution-processed $a$-IGZO TFTs under a negative gate-bias illumination stress (NBIS).

7. References. 


\section{Composition analysis of $a$-IGZO films with different annealing conditions.}

The chemical composition analysis of for the diversely treated $a$-IGZO films (1h DUV, 2h DUV, DW, DWD) was performed with X-ray photoelectron spectroscopy (XPS) analysis and Energy Dispersive Spectroscopy (EDS). Within typical error range of EDS and XPS, the chemical compositions from XPS and EDS showed identical atomic ratios for the same samples, which are proportional to molar concentration of indium, gallium, and zinc precursors (In: $0.085 \mathrm{M}, \mathrm{Ga}: 0.0125 \mathrm{M}, \mathrm{Zn}: 0.0275 \mathrm{M}){ }^{\mathrm{S} 1}$ The similar atomic ratios for entire $a$-IGZO samples confirm that the $a$-IGZO films maintained consistent chemical compositions during the diverse processing conditions, even after the water treatment.

\section{Table S1.}

\begin{tabular}{|c|c|c|c|c|c|c|}
\hline \multirow{2}{*}{ Simple } & \multicolumn{2}{|c|}{ In } & \multicolumn{2}{c|}{ Aa } & \multicolumn{2}{c|}{ An } \\
\cline { 2 - 8 } & XPS & EDS & XPS & EDS & XPS & EDS \\
\hline 1h DUV & 69.53 & 69.43 & 6.87 & 7.51 & 23.61 & 23.06 \\
2h DUV & 68.79 & 73.61 & 7.25 & 6.58 & 23.96 & 19.8 \\
DW & 69.26 & 72.62 & 7.58 & 9.62 & 23.16 & 17.76 \\
DWD & 69.39 & 73.7 & 7.34 & 7.38 & 23.27 & 18.92 \\
\hline
\end{tabular}

Table S1. Atomic composition ratio of $1 \mathrm{~h}$ DUV, $2 \mathrm{~h}$ DUV, DW, DWD-annealed $a$-IGZO films. 


\section{Electrical characterization of DUV-irradiated AIOx dielectric layer.}

The areal capacitance vs. frequency (C-F) and leakage current density vs. electric field (JE) characteristics of the DUV-annealed $\mathrm{AlO}_{\mathrm{x}}$ gate dielectric layer are shown in Figure $\mathrm{S} 1$. The areal capacitance of the DUV-annealed $\mathrm{AlO}_{\mathrm{x}}$ gate dielectric layer was $120 \mathrm{nF} \mathrm{cm}^{-2}$ at 100 $\mathrm{Hz}$, showing a dielectric constant of 5.42. However, the capacitance value slight decreases with increasing frequency $\left(104 \mathrm{nF} \mathrm{cm} \mathrm{cm}^{-2}\right.$ at $\left.1 \mathrm{MHz}\right)$ which can be attributed to structural defects such as hydroxyl group or oxygen vacancies in the film. ${ }^{\mathrm{S} 2}$ Meanwhile, the DUVannealed $\mathrm{AlO}_{\mathrm{x}}$ gate dielectric showed an excellent insulating property as shown Figure S1(b). Particularly, the DUV-annealed $\mathrm{AlO}_{\mathrm{x}}$ gate dielectric had a leakage current density of $7.14 \times 10^{-8} \mathrm{~A} \mathrm{~cm}^{-2}$ at $2 \mathrm{MV} \mathrm{cm}^{-1}$ and a breakdown field of $7.8 \mathrm{MV} \mathrm{cm}^{-1}$. Also, the distributions of leakage current density and dielectric breakdown field indicate that the DUV-annealed $\mathrm{AlO}_{\mathrm{x}}$ gate dielectric has reasonable spatial uniformity over the tested substrate [Figure $\mathrm{S} 1(\mathrm{c})$ ].
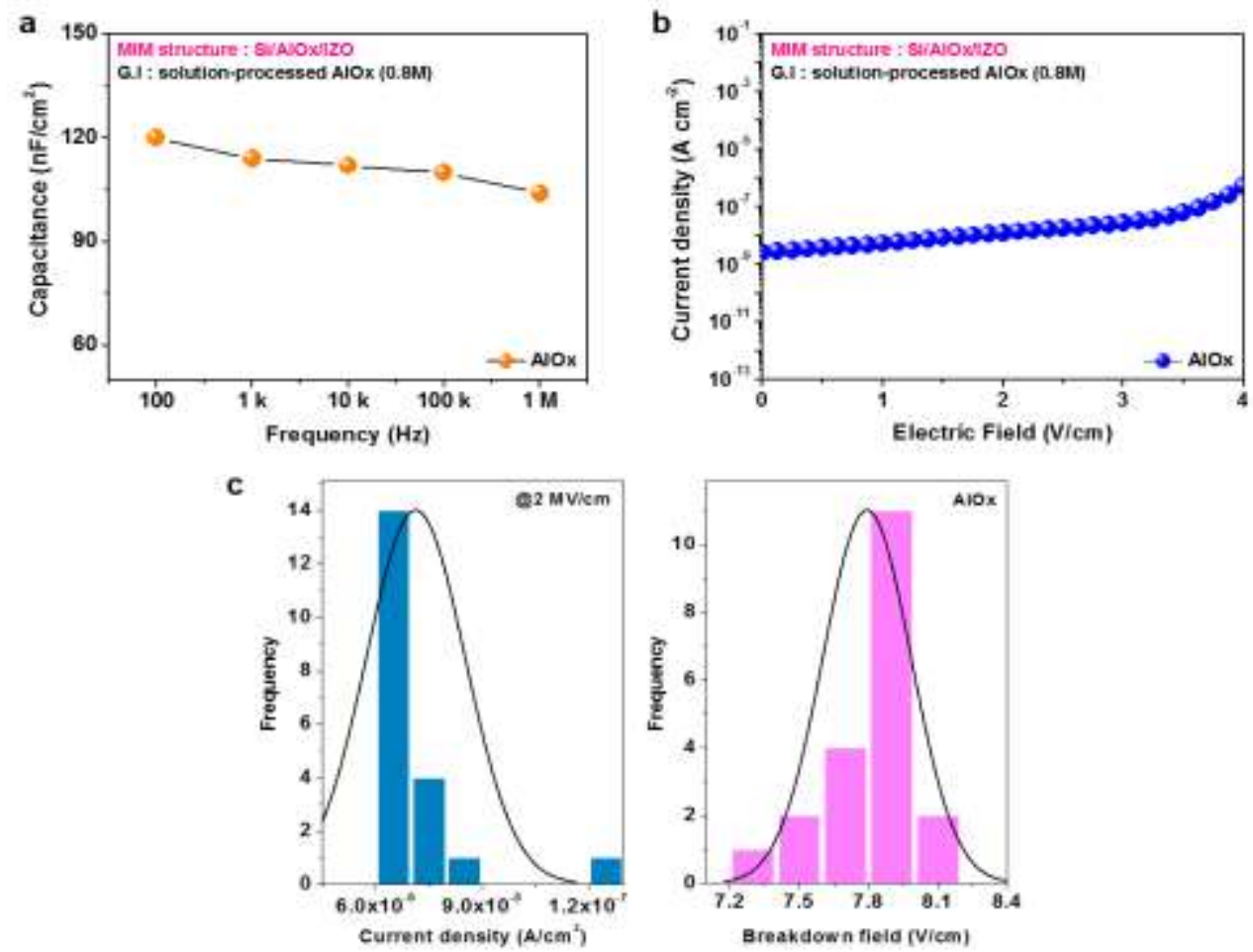

Figure S1. a, Capacitance per area-frequency (C-F) and b, Leakage current density-electric field (JE) of $\mathrm{AlO}_{\mathrm{x}}$ dielectric layer using M-I-M structure devices. c, Statistical distribution of current density and breakdown field of $\mathrm{AlO}_{\mathrm{x}}$ dielectric layer. 
3. The summarized electrical properties of DUV-irradiated AlOx dielectric layer.

Table S2.

\begin{tabular}{cccccc}
\hline Sample & $\begin{array}{c}\text { Breakdown field } \\
(\text { MV/cm) }\end{array}$ & $\begin{array}{c}\text { Current density } \\
\text { (at 2AV/cm) }\end{array}$ & $\begin{array}{c}\text { Capacitance } \\
\text { (at } 100 \mathrm{~Hz} \text { ) }\end{array}$ & Thickness & $\begin{array}{c}\text { Dielectric } \\
\text { Constant }\end{array}$ \\
\hline AlOx_DUV1h & 7.8 & $7.14 \times 10^{-8} \mathrm{~A} / \mathrm{cm}^{2}$ & $120 \mathrm{nF} / \mathrm{cm}^{2}$ & $40 \mathrm{~nm}$ & 5.42 \\
\hline
\end{tabular}

Table S2. The electrical characteristics of DUV-irradiated $\mathrm{AlO}_{\mathrm{x}}$ dielectric layer. 


\section{The transfer characteristics of DWD-annealed $a$-IGZO TFTs.}

For experimental simplicity, we used simple glass container with water spray as sample water treatment chamber. Therefore, the relative humidity of $70-80 \%$ for water treatment is not the optimized condition in this study. To investigate the water content effect, we tested different water content, 50-55\% RH and $100 \% \mathrm{RH}$ at $24 \pm 1{ }^{\circ} \mathrm{C}$, which corresponds to absolute water content of $\sim 12$ and $22 \mathrm{~g} / \mathrm{m}^{3}$. As shown in Figure S2, we do not observe any significant difference in device performance with water content increase. Compared with the water treated sample at $70-80 \% \mathrm{RH}$, the electrical properties of the water treated samples at both $50-55 \%$ RH and $100 \%$ RH show less than $10 \%$ difference.

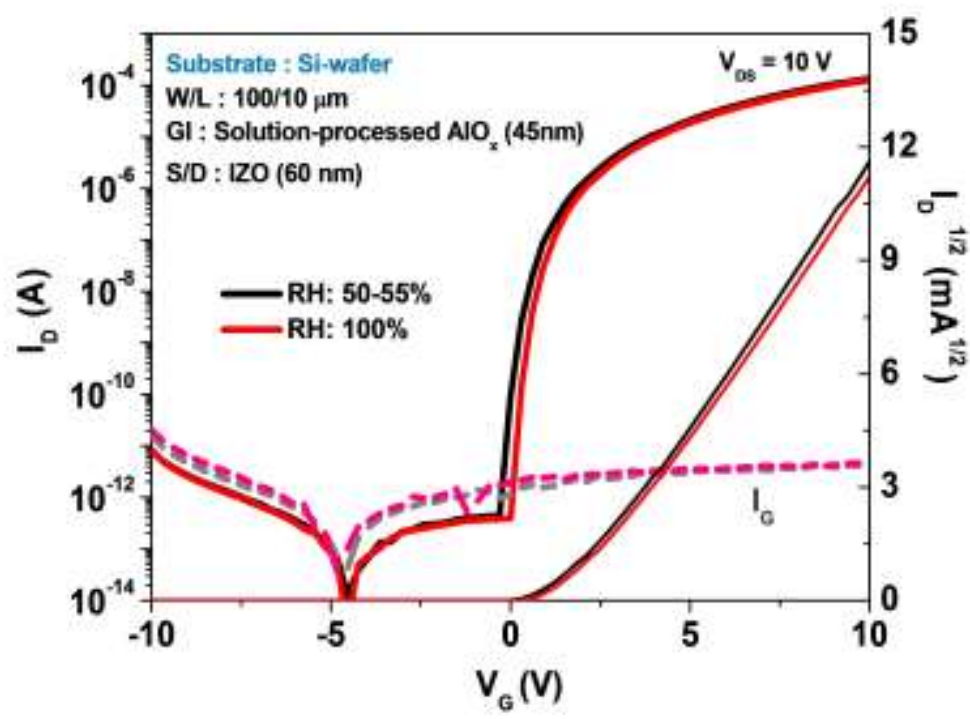

Figure S2. The transfer characteristics of DWD-annealed $a$-IGZO TFTs, which is stored at relative humidity of $50-55 \%$ and $100 \%$ for $1 \mathrm{~h}$. 


\section{The statistical distribution of hysteresis voltages.}

We have defined the difference of threshold voltages $\left(\mathrm{V}_{\mathrm{TH}}\right)$ obtained from forward gate sweep and reverse sweep current as hysteresis voltage. ${ }^{\mathrm{S} 3, \mathrm{~S} 4}$ The hysteresis level of our device is consistently quite small for all devices. The representative transfer plot shows similar minimized hysteresis within device-to-device variation range. The average hysteresis voltages of $1 \mathrm{~h}$ DUV-annealed, 2h DUV-annealed, DW-annealed, and DWD-annealed $a$ IGZO TFTs have $0.83,0.73,0.77$, and $0.72 \mathrm{~V}$, respectively.
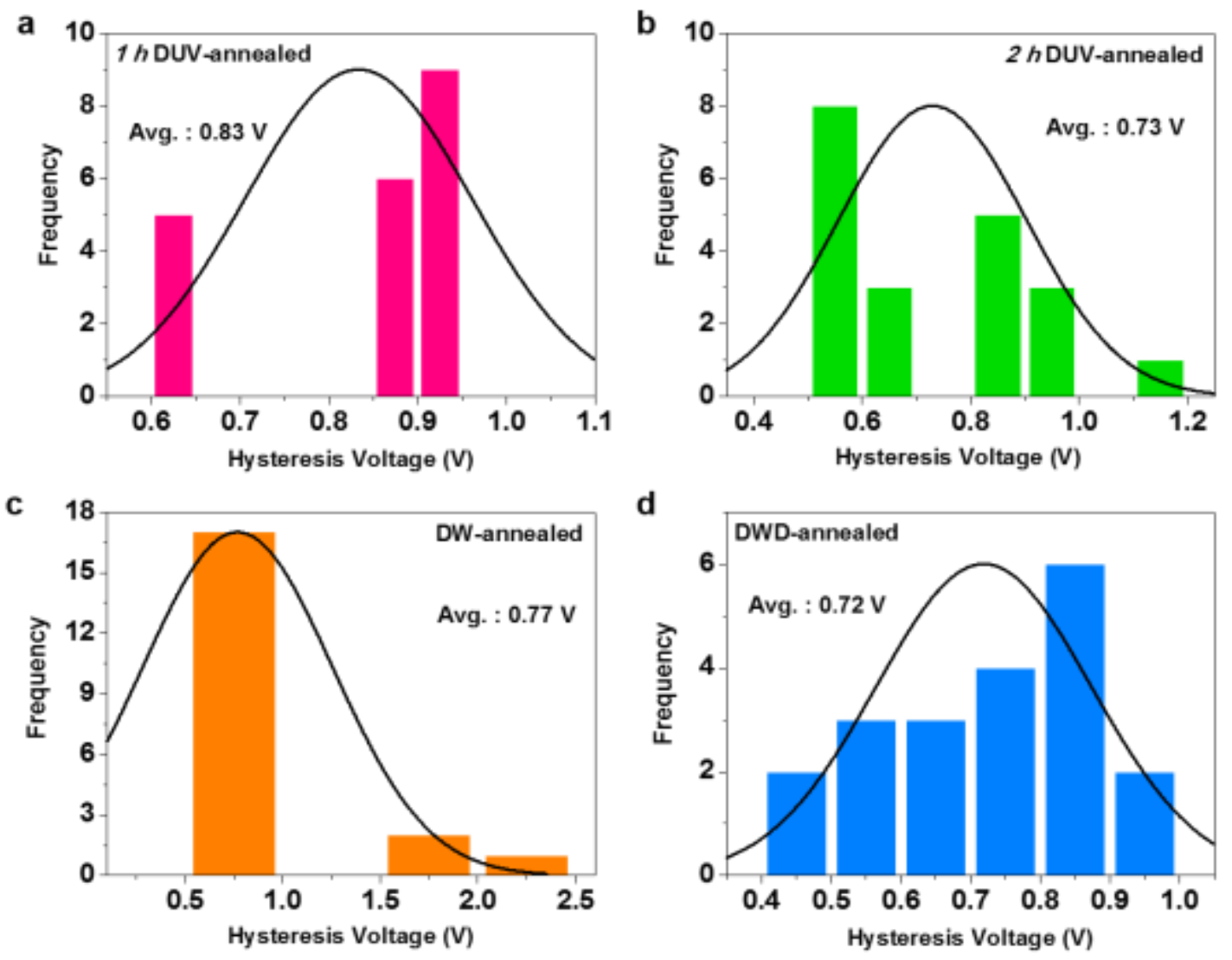

Figure S3. The statistical distribution of hysteresis voltages. a. $1 \mathrm{~h}$ DUV-annealed, b. $2 \mathrm{~h}$ DUVannealed, c. DW-annealed, and d. DWD-annealed $a$-IGZO TFTs. 


\section{The threshold voltage shift of solution-processed $a$-IGZO TFTs under NBIS.}
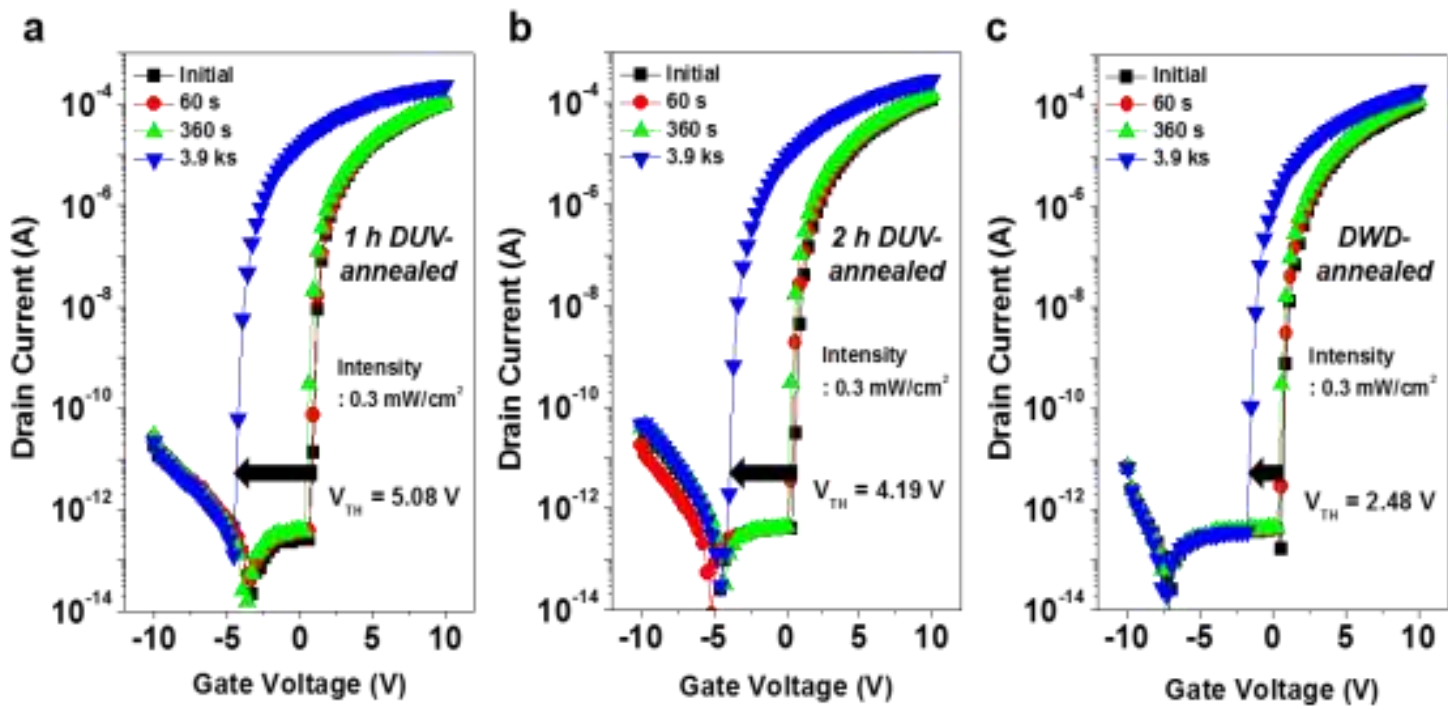

Figure S4. Threshold voltage shift of solution-processed $a$-IGZO TFTs under a negative illumination gate-bias stress (NBIS) ( $\mathrm{V}_{\mathrm{GS}}=-5 \mathrm{~V}, \mathrm{~V}_{\mathrm{DS}}=+0.1 \mathrm{~V}, \mathrm{t}=3,900 \mathrm{sec}$ ). (a) $1 \mathrm{~h}$ DUV-annealed, (b) $2 \mathrm{~h}$ DUV-annealed, and (c) DWD-annealed $a$-IGZO TFTs. 


\section{References}

(S1) Waldo, R. A.; Militello, M. C.; Gaarenstroom, S. W. Quantitative Thin-Film Analysis with an Energy-Dispersive X-ray Detector. Surf. Interface. Anal. 1993, 20.2: 111-114.

(S2) Park, J. H.; Kim, K.; Yoo, Y. B.; Park, S. Y.; Lim, K.-H.; Lee, K. H.; Baik, H. K.; Kim, Y. S. Water Adsorption Effects of Nitrate Ion Coordinated A12O3 Dielectric for High Performance Metal-Oxide Thin-Film Transistor. J. Mater. Chem. C 2013, 1 (43), 7166-7174

(S3) Qu, M.; Li, H.; Liu, R.; Zhang, S.-L.; Qiu, Z.-J. Interaction of Bipolaron with the H2O/O2 Redox Couple Causes Current Hysteresis in Organic Thin-Film Transistors. Nat. Commun. 2014, 5, 3185.

(S4) Yang, M. H.; Teo, K. B. K.; Gangloff, L.; Milne, W. I.; Hasko, D. G.; Robert, Y.; Legagneux, P. Advantages of Top-Gate, High-K Dielectric Carbon Nanotube Field-Effect Transistors. Appl. Phys. Lett. 2006, 88 (11), 2004-2007. 